\title{
diagrama de aumento de resistencia y resistencia de referencia
}

\section{1. diagrama de aumento de la resistencia [diagrama $F \mathbf{Z}$ ]}

$\mathrm{El}$ diagrama $\mathrm{FZ}$ ha resultado ser un medio auxiliar muy eficaz en la representación del aumento de resistencia de un cemento. El principio de la representación de tal diagrama consiste en que no se toma como abscisa el tiempo, sino que se elige una resistencia de referencia, representando en dirección perpendicular a ella, y hacia abajo, L liferencia frente al valor anterior, y la diferencia frente al valor siguiente, hacia arriba. a transformación del diagrama corriente de resistencia/tiempo en un diagrama FZ a avés de la resistencia de referencia después de 7 días se muestra en la figura 1.

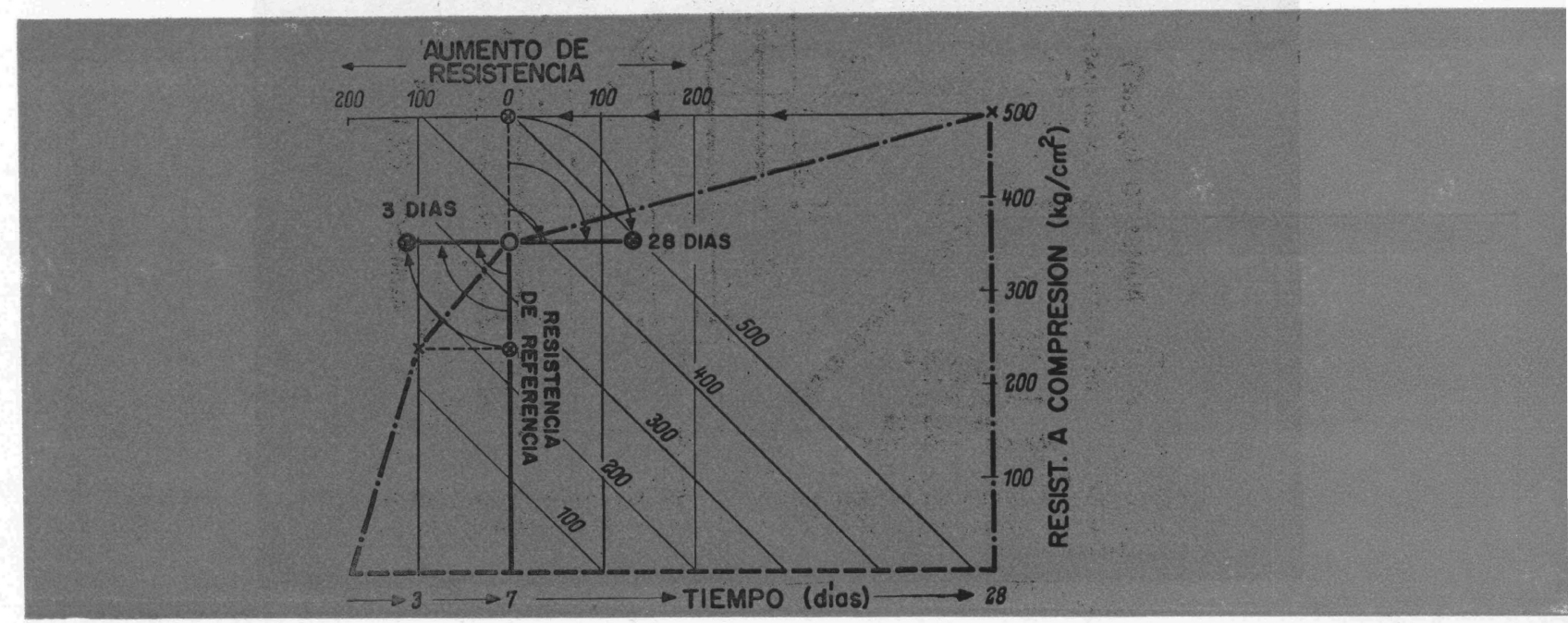

Fig. 1.-Diagrama de resistencia-tiempo y diagrama del aumento de resistencia referido a la resistencia a 7 días.

En la figura 2 se han representado en dicho diagrama, sobre la abscisa, los valores de tres días como resistencias de referencia, valores medios de una serie de cementos portland $(\mathrm{P})$, cementos ferroportland $(\mathrm{E})$, cementos siderúrgicos $(\mathrm{H})$ de la calidad $\mathrm{Z} 375$ y de una serie de cementos portland de la calidad Z 475, así como un grupo de representantes típicos de esta categoría. 


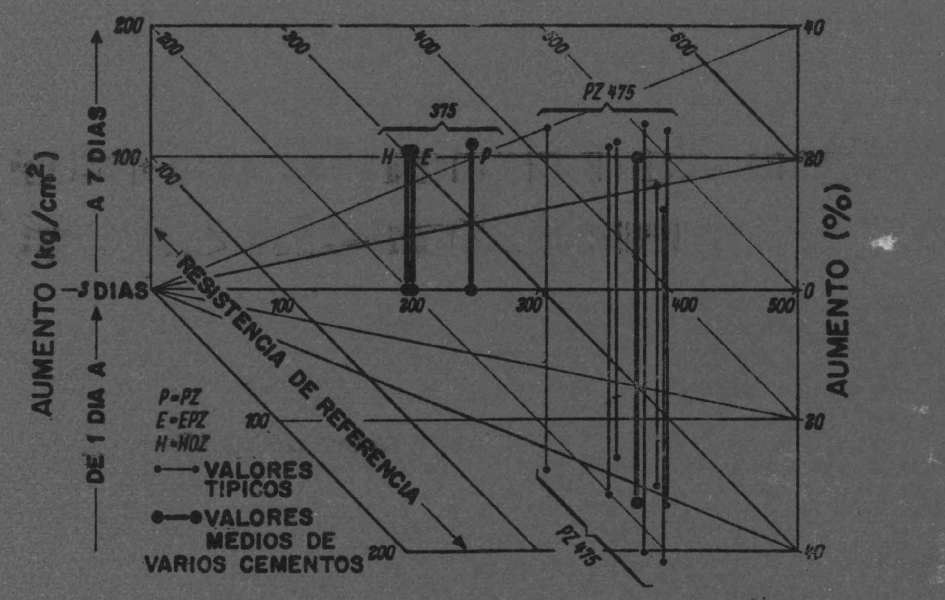

Fig. 2. - Diagrama de aumento de resistencia a compresión a 1,3 y 7 dias para $Z 375$ y $Z 475$, referido a la resistencia a 3 días.

En sentido perpendicular, y en la parte superior de la abscisa, se ha representado el aumento de resistencia, o sea, la diferencia frente a la resistencia del siguiente ensayo después de 7 dias; en el último grupo de cementos se han representado también las diferencias frente a la resistencia del ensayo anterior, es decir, la resistencia a 1 día.

En la figura 3 se hizo lo mismo con las resistencias después de 3,7, y 28 días, empleando la resistencia a 7 dias como referencia. La serie de las resistencias representadas pudo ampliarse en este caso con valores de una serie de cementos de la calidad Z 275.

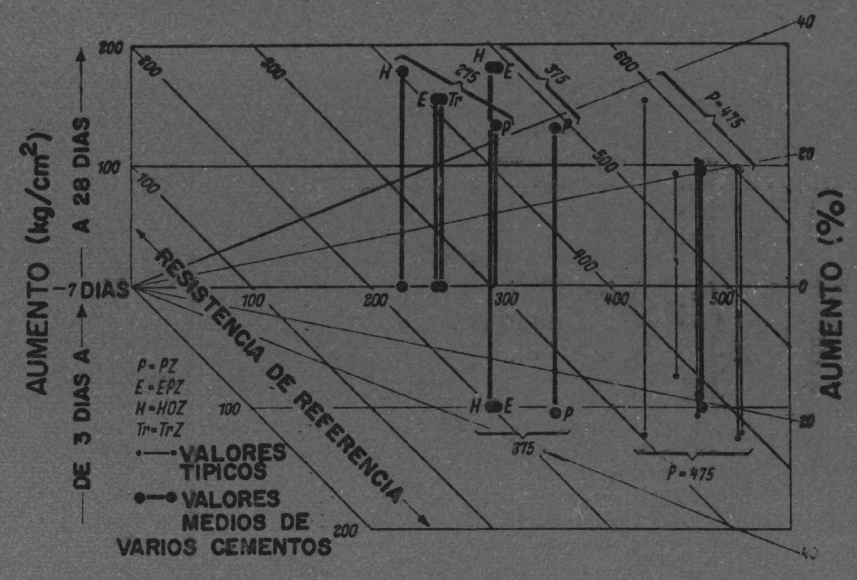

Fig. 3.-Diagrama de aumento de resistencia después de 3,7 y 28 dias, para $? 275, Z 375$ y $Z 475$, referido a la resistencia a 7 dias.

La forma de representación con segmentos facilita la comparación de los cementos según sus valores de aumento, tanto en $\mathrm{kg} / \mathrm{cm}^{2}$ como en $\%$, en función de la resistencia de referencia (ordenadas izquierda y derecha). Si se introducen en el diagrama coordenadas a $45^{\circ}$ pueden leerse también las resistencias anterior y posterior expresadas en $\mathrm{kg} / \mathrm{cm}^{2}$. Se ven claramente las características típicas de las distintas clases de cemento y las diferentes calidades debidas a su composición y a la finura de molienda. Llama la atención el aumento de resistencia idéntico de todos los cementos de 3 a 7 días, que asciende a unos 
$100 \mathrm{~kg} / \mathrm{cm}^{2}$, según se ve en las figuras 2 (parte superior) y 3 (parte inferior). Pero esto significa un aumento de más del $40 \%$ en los cementos de la calidad $Z 375$, como indican las rayas que parten del punto $O$ para el aumento de porcentaje, mientras que en los cementos de la calidad Z 475 está generalmente por debajo. La figura 3, en su parte superior, expresa claramente que el aumento de resistencia de 7 a 28 días en la misma clase de cementos (P, E o H) es casi igual, es decir, en el caso de cementos portland más o menos de $150 \mathrm{~kg} /$ $\mathrm{cm}^{2}$; o sea, aproximadamente del $40 \%$, y en el caso de cementos de alto horno aún mayor. Con esto tiene lugar la adaptación de los valores finales de 28 días para las tres clases de cemento P, E y H dentro de las dos clases de calidad $\mathrm{Z} 275$ y Z 375, que se ve claramente por la situación a $45^{\circ}$ de sus valores finales.

\section{2. comparación hecha hasta ahora de las caracteristicas hidráulicas y puzolánicas}

EI diagrama FZ es especialmente apropiado para la representación del efecto de materias puzolánicas, para las cuales, como se sabe, no hay todavía una valoración apropiada. Una solución parcial sería valorar los índices puzolánicos por determinación de la fijación de cal, pero los fabricantes de cemento y los consumidores del mismo preferirán siempre conocer las variaciones de la resistencia. Una puzolana que fija la cal, pero que disminuye la resistencia, no es interesante, y las ventajas y los inconvenientes de una fijación de cal serán siempre objeto de discusiones. Por eso se debe intentar siempre fijar lo más claramente posible la posición de la resistencia de mezclas de cemento portland con distintas materias hidráulicas y puzolánicas.

La determinación del índice hidraúlico es satisfactoria casi exclusivamente en las arenas siderúrgicas. Los índices hidráulicos dependen de muchos factores. En un caso extremo, por ejemplo con ensayos de 3 a 20 días y mezclas con un contenido de por lo menos el $40 \%$, su valor oscila:

- en el caso de la arena siderúrgica, entre 28 y $62 \%$;

- en el caso de la puzolana, entre 17 y $48 \%$.

Probablemente, el error fundamental en la determinación del índice hidráulico reside en que se basa en la suposición de que la harina de cuarzo es inerte, no importando si ha sido molida a la finura del cemento - unos $3.000 \mathrm{~cm}^{2} / \mathrm{g}-\mathrm{o}$, como en este caso, a la finura del trass (puzolana) - unos $5.000 \mathrm{~cm}^{2} / \mathrm{g}$-.Probablemente reaccione también con la cal del cemento endurecido, aparte de tener sus efectos de relleno y refuerzo.

\section{3. la resistencia comparativa como resistencia de referencia}

Como resistencia de partida debería tomarse como base otro valor que no fuese la resistencia de la mezcla de clínker y cuarzo. Por esta razón se recurrió a la resistencia comparativa, que se funda solamente en la resistencia de un cemento portland puro, es decir, 
un solo välor. La resistencia comparativa es una magnitud de cálculo que corresponde al contenido correspondiente de cemento portland y asciende, por ejemplo, a un $85 \%$ del valor del cemento portland puro, si se ha sustituido el $15 \%$ de dicho cemento portland. De esta forma la valoración del comportamiento de la arena de cuarzo queda independiente y se conoce su papel en comparación con las demás sustancias añadidas.

En la figura 4 se muestra cómo puede derivarse de una representación corriente, situada en la parte inferior de la figura 4, el diagrama $\mathrm{FZ}$, de la parte superior de la misma figura, para mezclas de clínker de cemento con un 15, 30 y $50 \%$ de cuarzo Q, puzolana P y arena siderúrgica H. En la parte inferior de la figura 4 se ha expresado el cambio de resistencia para $\mathrm{H}, \mathrm{P}$ y $\mathrm{Q}$, en relación con el contenido creciente del 15,30 y 50 e incluso $70 \%$, o sea, los valores de ensayo a la edad de 28 días según el procedimiento RILEMCEMBUREAT.
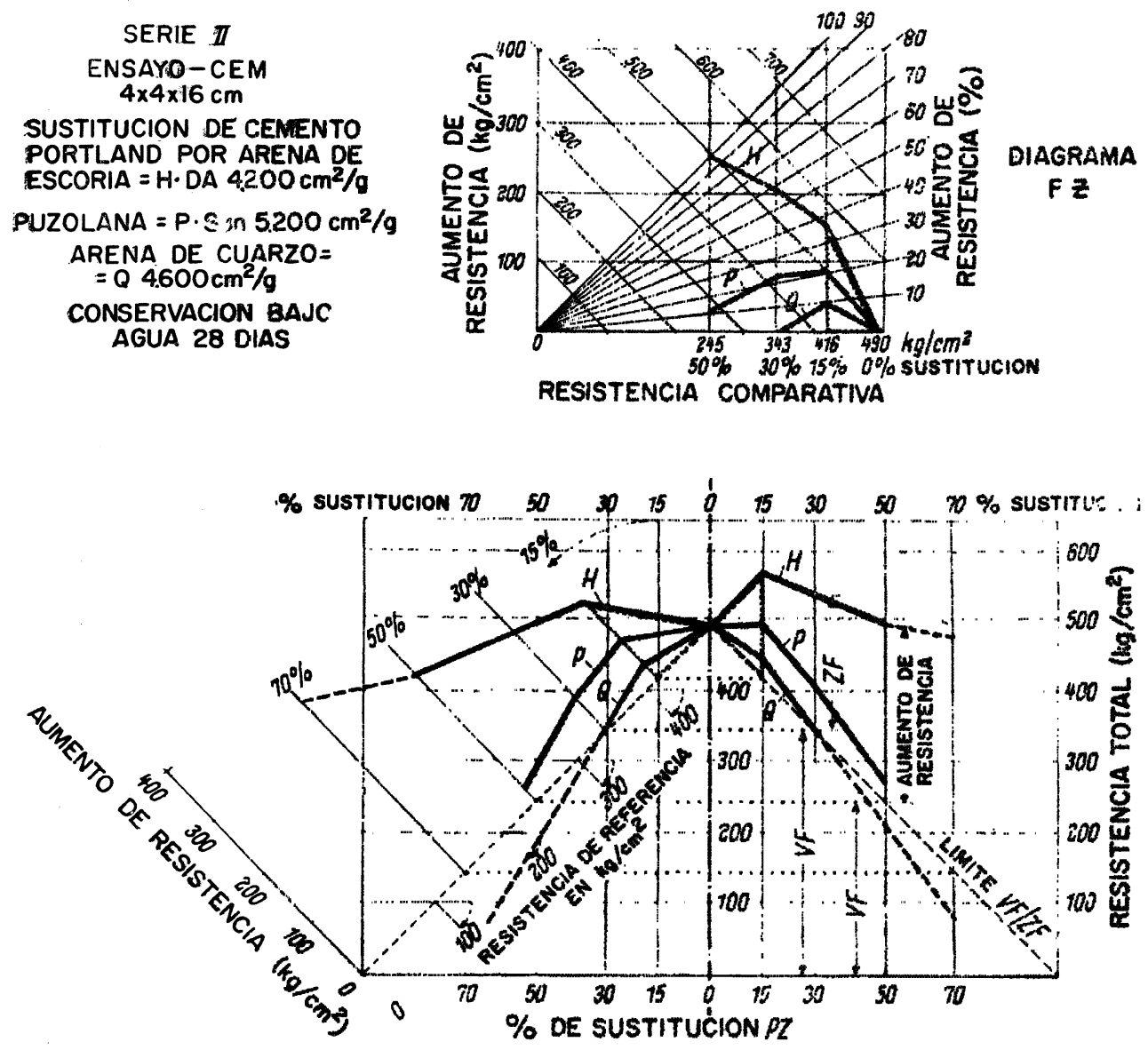

Fig. 1. Nitodo de obtencion hel diagrama de resistencia.

Se ve claramente el doble pape! s! la harina lina de cuarzo que desarrolla, en primer lugar, su función como fomentador $\mathrm{s}$, en scigundo lugar. su función coma reducter de la resistencia. Basta trazal uma linea desde el rator de rotura del cementu porlland (190 kilogramos $/ \mathrm{em}^{2}$ ), a través del punlo (ero de la abstisa (centro del dibujo inferion), hasta el punto $100 \%$ de la abscirit. donde no puede existir ya ninguna resistencia hidráulica. 
Esta línea corta los segmentos perpendiculares de $\mathrm{H}, \mathrm{P}$ y Q, cuya parte superior es el aumento de resistencia ZF. Debajo se encuentra la resistencia comparativa VF, que también puede calcularse a partir del producto $490 \times 85 \%=416 \mathrm{~kg} / \mathrm{cm}^{2}$ para una sustitución del $15 \% ; 490 \times 70 \%=343 \mathrm{~kg} / \mathrm{cm}^{2}$ para el $30 \%$, y $490 \times 50 \%=245 \mathrm{~kg} / \mathrm{cm}^{2}$ para el $50 \%$. Si ahora se coloca el aumento de resistencia ZF por encima de la resistencia comparativa VF y se dobla al mismo tiempo hacia la izquierda la parte derecha de la representación, empleando como eje la línea límite $\mathrm{VF} / \mathrm{ZF}$ de la izquierda, se obtiene el diagrama ZF sobre la resistencia comparativa o de referencia. En la parte superior de la figura 4, la abscisa de la resistencia comparativa está en situación horizontal. Ya se había añadido el haz de líneas a $45^{\circ}$ para leer la resistencia total, y las lineas que parten del punto cero, para conocer el aumento en el tanto por ciento de la resistencia.

Se obtiene una ventaja muy grande con el cambio de las curvas de aumento de la resistencia para distintas edades, por ejemplo después de 7, 28 y 90 días. Con esto se puede representar al mismo tiempo todas las edades como se hace en la figura 5 para cuarzo $Q$, piedra caliza $\mathrm{K}$ y el campo rayado de 4 puzolanas.

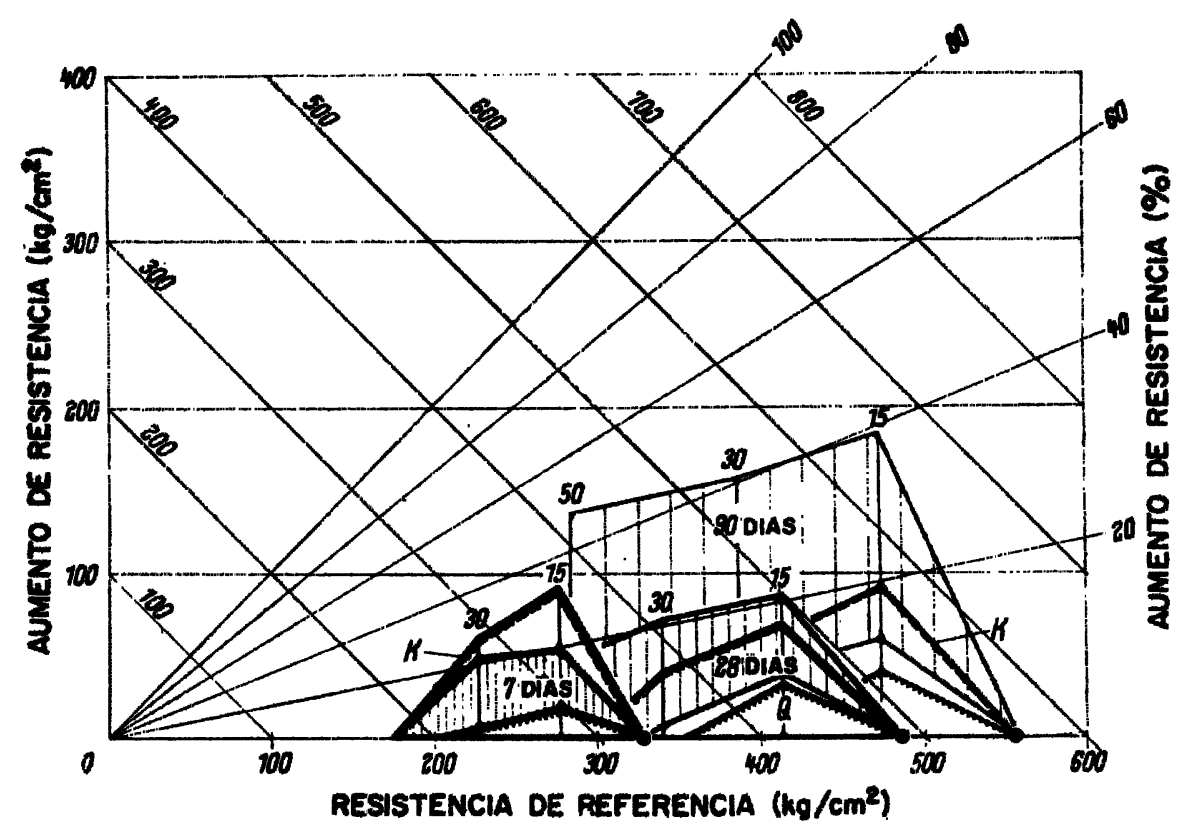

Fig. 5.-Diagrama de aumento de resistencia, Sustitución de 15,30 y $50 \%$ de $17 \%$ con puzolana 4, caliza y cuarzo molido (K, Q). Probetas de mortero CE.II, $4 \times$ $\times 4 \times 16 \mathrm{~cm}$, curadas en agua durante 7,28 y 90 dias.

Sobre el valor objetivo de esta figura y de la anterior no queremos decir en este artículo otra cosa que lo expresado por la altura y la longitud de las curvas de aumento; en la hidraulicidad tiene validez el orden siguiente: cuarzo, piedra caliza, puzolana, arena siderúrgica, clínker de cemento.

Con este método de representación el campo de las 4 puzolanas a la edad de 7 días está situado todavía dentro de las sustancias inertes (cuarzo y piedra caliza) colocándose sólo al cabo de 90 días casi totalmente por encima de la zona de la piedra caliza. Con esto no se quiere decir nada de si pueden existir tales curvas de aumento en el caso de hormigón corriente y hasta qué punto. 
En este momento se está realizando la valoración de todas las experiencias con otras adiciones, así como de otros procedimientos de ensayo, que se hicieron en colaboración con H. Mathieu y que se publicarán más adelante. Con esta comunicación anticipada queríamos señalar solamente la posibilidad de representar una serie de resistencias en su sucesión normal en el tiempo sin considerar éste y hacer conocer el efecto de sustancias hidráulicas y puzolánicas en procedimientos de ensayo corrientes con la meta de valorarlos numéricamente.

\section{resumem}

El diagrama de aumento de la resistencia ha demostrado ser un medio apropiado para la representación gráfica del crecimiento de la resistencia de un cemento. Cuando este modo de representación se aplica a cementos en los que una parte del clínker se ha reemplazado por arena de alto horno, puzolana, caliza o cuarzo, se obtiene un gráfico que demuestra claramente las modificaciones aportadas en las resistencias de estas mezclas por la sustitución.

Lo esencial de la representación mediante el diagrama de aumento de resistencia reside en el hecho de que en abscisas no se lleva el tiempo, sino una resistencia de referencia. En la vertical de cada valor se inscribe la diferencia con el valor precedente hacia abajo y la diferencia con el valor siguiente hacia arriba. En el diagrama de aumento de resistencia destinado a representar los efectos de aumento o disminución de resistencia aportados por ciertos aditivos se elige, como eje de la resistencia de referencia, la resistencia del cemento portland puro y la resistencia de referencia calculada a partir del contenido en cemento portland. De esta forma, la influencia sobre la resistencia de cemento de los materiales hidráulicos y de la puzolana se reconocen claramente bajo forma de curvas de crecimiento.

Los ensayos, que han sido hechos en colaboración con II. Mathieu, se publicarán en un artículo posterior. 